\title{
Effect of baryon density on parton production, chemical equilibration and thermal photon emission from quark gluon plasma
}

\author{
D. Dutta, A. K. Mohanty, K. Kumar and R. K. Choudhury \\ Nuclear Physics Division, Bhabha Atomic Research Centre, Mumbai, India-400085.
}

(February 23, 2018)

\begin{abstract}
The effect of baryon density on parton production processes of $g g \rightleftharpoons g g g$ and $g g \rightleftharpoons q \bar{q}$ is studied using full phase space distribution function and also with inclusion of quantum statistics i.e. Pauli blocking and Bose enhancement factors, in the case of both saturated and unsaturated quark gluon plasma. The rate for the process $g g \rightleftharpoons q \bar{q}$ is found to be much less as compared to the most commonly used factorized result obtained on the basis of classical approximation. This discrepancy, which is found both at zero as well as at finite baryon densities, however, is not due to the lack of quantum statistics in the classical approximation, rather due to the use of Fermi-Dirac and BoseEinstein distribution functions for partons instead of Boltzmann distribution which is appropriate under such approximation. Interestingly, the rates of parton production are found to be insensitive to the baryo-chemical potential particularly when the plasma is unsaturated although the process of chemical equilibration strongly depends on it. The thermal photon yields, have been calculated specifically from unsaturated plasma at finite baryon density. The exact results obtained numerically are found to be in close agreement with the analytic expression derived using factorized distribution functions appropriate for unsaturated plasma. Further, it is shown that in the case of unsaturated plasma, the thermal photon production is enhanced with increasing baryon density both at fixed temperature and fixed energy density of the quark gluon plasma.
\end{abstract}

PACS number(s):25.75.Dw, 12.38.Mh, 12.38.Bx, 24.85.+p

Submitted to Phys. Rev. C 


\section{INTRODUCTION}

The future collider experiments with heavy ions will provide an opportunity to investigate the physics of strongly interacting matter at extreme energy densities where the formation of a new state of matter, the quark gluon plasma (QGP), is expected. With these colliders, it may be possible to achieve energy density well above the deconfinement threshold to be able to probe the QGP in its asymptotically free 'ideal gas' form. At high energies, the central rapidity region may have nearly vanishing baryon density, similar to the early universe, and will probably consist of a very dense system of semi-hard partons (mini-jets) which would lead to rapid thermalization and extremely high initial temperature [四]. In the standard scenario, the quark gluon plasma formed during the collision is expected to thermalise in a typical time scale of $(\approx 1 \mathrm{fm} / \mathrm{c})$ and the subsequent evolution follows the ideal Bjorken's scaling solution [2]. Several theoretical calculations based on perturbative QCD approaches now suggest that the partonic fluid which is mostly gluonic will attain kinetic equilibrium after a proper time $\tau_{0} \approx 0.1-0.3 \mathrm{fm} / \mathrm{c}$ [3]. This is justified given the fact that the rate of elastic collisions between quarks and gluons which establish thermal and mechanical equilibrium (referred as kinetic equilibrium corresponding to isotropic momentum distribution) is much larger than for inelastic collisions which establish chemical equilibrium. The remaining question is whether the high energy density matter remains in QGP phase sufficiently long enough to actually reach chemical equilibrium so that it can be identified with the QGP phase seen in lattice QCD calculations.

In the framework of pQCD based models, it is now understood that the densities of quarks and anti-quarks may stay well below the gluon density and may not reach up to the full equilibration value as required by an ideal chemical mixture of quarks and gluons [3]. Because of the consumption of energy by additional parton production, the temperature of the parton plasma falls down faster than the ideal solution where $T^{3} \tau$ remains constant. Essentially, same picture emerges in a somewhat different approach first proposed by Biro

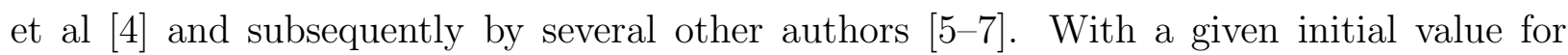
the energy and number densities, this type of calculations employ ideal fluid dynamics to study the subsequent evolution of the kinetically equilibrated QGP phase coupled to rate equations to determine the chemical composition.

As mentioned before, the evolution of the partonic matter toward a fully (chemically) equilibrated QGP will be dictated by the parton proliferation mainly through induced ra- 
diation and gluon fusion [4,8]. Unfortunately, there have been no rigorous thermal field theoretical calculations for the rate of quark production since the basic cross section diverges for the mass less quarks. The closest approach to a full calculation has been done by using the thermal quark mass and Debye screening mass to regularize the divergence [4.9]. However, as used by Biro et al [4] and subsequently by many others [5 [7], the quark production rate $g g \rightleftharpoons q \bar{q}$ has been factorized using classical approximation where Pauli blocking and Bose enhancement factors (effect of quantum statistics) have been eliminated. The calculations have also been simplified further by assuming an ideal baryon free plasma. There is now enough evidence from theoretical studies that even at RHIC energies, the mid rapidity region may not be completely baryon free [10].

In an earlier work, we had studied chemical equilibration of the QGP at finite baryon density [7] where we had considered the processes $g g \rightleftharpoons g g g$ and $g g \rightleftharpoons q \bar{q}$ as the two dominant channels contributing to parton production. It is natural to think that the presence of baryon density $(\mu)$ in quark and anti-quark distribution functions may directly affect the process $g g \rightleftharpoons q \bar{q}$, whereas the process $g g \rightleftharpoons g g g$ may depend on $\mu$ only through the Debye screening mass used to regularize the divergence. Using modified Fermi-Dirac distribution function for quarks (anti-quarks) and a factorized distribution function for gluons valid at small baryon density, it was found in the above work that the two rates are not sensitive to the baryo-chemical potential particularly when the plasma is unsaturated. Therefore, we had used the same factorization (for $g g \rightleftharpoons q \bar{q}$ ) as used by Biro et al in the case of baryon free plasma. Although, the parton production rates for unsaturated plasma are not very sensitive to baryo-chemical potential, the energy, quark and anti-quark densities (hence the rate of chemical equilibration) strongly depend on $\mu$. It was also shown that due to chemical equilibration, the baryon rich plasma cools at a slower rate as compared to the baryon free plasma.

In the present work, we have studied the role of baryon density on parton production particularly for the process $g g \rightleftharpoons q \bar{q}$ in detail using full phase space distribution functions for partons and also including Pauli blocking and Bose enhancement factors explicitly. Specifically, we investigate the effect of quantum statistics particularly in the presence of finite baryon density. The present calculations show that the rate for the process $g g \rightleftharpoons q \bar{q}$ is lowered by $20 \%$ to $30 \%$ as compared to the factorized result of Biro et al as given in Ref. 四. This discrepancy, however, is not due to the lack of quantum statistics, but rather due to the use of Fermi-Dirac (FD) and Bose-Einstein (BE) type distribution functions for quarks and gluons in Ref. [4] instead of Boltzmann distribution which would have been appropriate under classical approximation. With the revised rates, we have also studied the process of 
chemical equilibration solving a set of rate equations. The quark and anti-quark fugacities slow down whereas the gluon fugacity goes up slightly as compared to the previous values. However, there is no net effect on the evolution of temperature which remains practically unchanged. Further, it is shown that the rates of parton production are insensitive to the presence of baryon density particularly when the plasma is unsaturated. The findings of the present study are also consistent with our earlier work [7]. We had earlier studied the thermal dilepton yields as a probe of QGP. We investigate here the thermal hard photon production as a complementary probe from a chemically non-equilibrated baryon rich quark gluon plasma. It is seen that, like the dilepton yields, the photon yield is also enhanced in presence of baryon density.

The paper is organized as follows. In section II, we calculate the parton production and chemical equilibration at finite baryon density. In section III, we calculate the thermal photon yield from chemically unsaturated plasma both numerically and analytically. The results have been summarized in section IV.

\section{PARTON PRODUCTION AND CHEMICAL EQUILIBRATION}

In this section, we calculate the thermal production rates for (i) gluon fusion $g g \rightleftharpoons q \bar{q}$ and (ii) gluon radiation $g g \rightleftharpoons g g g$. We also study the process of chemical equilibration by solving the ideal fluid hydrodynamics coupled to a set of rate equations. We use the Juttner distribution functions for quarks (anti-quarks) and gluons as given by

$$
f_{q}(\bar{q})=\frac{\lambda_{q}(\bar{q})}{\lambda_{q}(\bar{q})+e^{(p \mp \mu) / T}}=\frac{\lambda_{q(\bar{q})} e^{ \pm x}}{\lambda_{q(\bar{q})} e^{ \pm x}+e^{p / T}} \quad ; \quad f_{g}=\frac{\lambda_{g}}{e^{p / T}-\lambda_{g}}
$$

where $x=\mu / T$. The fugacity factor $\lambda_{i}(i=q, \bar{q}$ and $\mathrm{g})$ gives the measure of the deviation of the distribution functions from the equilibrium values and $\mu\left(=\mu_{B} / 3\right)$ is the quark-chemical potential. The chemical equilibrium is said to be achieved when $\lambda_{i} \rightarrow 1$. As mentioned in Ref. [7], the quark (anti-quark) distribution functions could also be written using the commonly used definition of fugacities $\lambda_{Q}$ and $\lambda_{\bar{Q}}$ given by

$$
\lambda_{Q(\bar{Q})}=e^{ \pm x} \lambda_{q(\bar{q})}
$$

so that, there is no need to use the quark chemical potential $\mu$ explicitly. However, the definition (1) is quite convenient, since at equilibrium where $\lambda_{q}=\lambda_{\bar{q}}=1$, the chemical potential associated with $\lambda_{i}$ vanishes but the baryo-chemical potential still exists. Further, we have taken $\lambda_{q}=\lambda_{\bar{q}}$ at all values of $\tau$ so that when $\mu \rightarrow 0, \lambda_{Q}=\lambda_{\bar{Q}}=\lambda_{q}$ resulting in a 
baryon symmetric matter. While we calculate the parton production rates using the above distribution functions, we also consider the approximations for quarks (anti-quarks) as

$$
f_{q(\bar{q})}=\frac{\lambda_{q(\bar{q}} e^{ \pm x}}{\lambda_{q(\bar{q})} e^{ \pm x}+e^{p / T}} \approx \frac{\lambda_{q(\bar{q})} e^{ \pm x}}{1+e^{p / T}}=\lambda_{q(\bar{q})} e^{ \pm x} f_{q(\bar{q})}^{e q}
$$

and for gluon distribution function as

$$
f_{g}=\lambda_{g} f_{g}^{e q}
$$

where $f_{q(\bar{q})}^{e q}=\left(1+e^{p / T}\right)^{-1}$ and $f_{g}^{e q}=\left(e^{p / T}-1\right)^{-1}$. The approximation given by Eq. $(3)$ is referred as modified Fermi-Dirac (MFD) distribution in Ref. [7] which becomes Fermi-Dirac distribution when $\lambda_{Q(\bar{Q})} \rightarrow 1$. Hence forth, we will refer both Eqs.(3) and (4) as modified factorized distributions (MFD) which are different from Boltzmann (BM) type of factorized form given by,

$$
f_{q}(\bar{q})=\lambda_{q}(\bar{q}) e^{-(p \mp \mu) / T} ; \quad f_{g}=\lambda_{g} e^{-p / T}
$$

\section{A. Gluon fusion}

We begin by calculating the rate for the process $g g \rightleftharpoons q \bar{q}[\mathbb{Q}]$ :

$$
\begin{gathered}
R_{g \rightarrow q}=\frac{1}{2} \int \frac{d^{3} p_{1}}{(2 \pi)^{3} 2 E_{1}} \int \frac{d^{3} p_{2}}{(2 \pi)^{3} 2 E_{2}} \int \frac{d^{3} p_{3}}{(2 \pi)^{3} 2 E_{3}} \int \frac{d^{3} p_{4}}{(2 \pi)^{3} 2 E_{4}}(2 \pi)^{4} \delta^{4}\left(p_{1}+p_{2}-p_{3}-p_{4}\right) \times \\
\sum\left|\mathcal{M}_{g g \rightarrow q \bar{q}}\right|^{2} f_{g}\left(p_{1}\right) f_{g}\left(p_{2}\right)\left[1-f_{q}\left(p_{3}\right)\right]\left[1-f_{\bar{q}}\left(p_{4}\right)\right]
\end{gathered}
$$

and

$$
\begin{gathered}
R_{q \rightarrow g}=\frac{1}{2} \int \frac{d^{3} p_{1}}{(2 \pi)^{3} 2 E_{1}} \int \frac{d^{3} p_{2}}{(2 \pi)^{3} 2 E_{2}} \int \frac{d^{3} p_{3}}{(2 \pi)^{3} 2 E_{3}} \int \frac{d^{3} p_{4}}{(2 \pi)^{3} 2 E_{4}}(2 \pi)^{4} \delta^{4}\left(p_{1}+p_{2}-p_{3}-p_{4}\right) \times \\
\sum\left|\mathcal{M}_{q \bar{q} \rightarrow g g}\right|^{2} f_{q}\left(p_{3}\right) f_{\bar{q}}\left(p_{4}\right)\left[1+f_{g}\left(p_{1}\right)\right]\left[1+f_{g}\left(p_{2}\right)\right]
\end{gathered}
$$

In Eq.(6), the squared matrix element, summed over spin and color, $\sum|\mathcal{M}|^{2}$ is weighted by two gluon distribution functions $f_{g}$ for the initial states. The factor $\left[1-f_{q}\right]\left[1-f_{\bar{q}}\right]$ indicates Pauli blocking for the final states. In the reverse process Eq.(7)), the rate is weighted by the distribution functions of quarks and anti-quarks for the initial states and the gluon final states each gain an enhancement factor $\left[1+f_{g}\right]$ due to Bose-Einstein statistics. The factor of $1 / 2$ accounts for the identity of the two gluons. Using the identity that results from Eq.(1), 


$$
\left[1-f_{q(\bar{q})}\right]=\frac{f_{q(\bar{q})}}{\lambda_{Q(\bar{Q})}} e^{p / T} ; \quad\left[1+f_{g}(p)\right]=\frac{f_{g}}{\lambda_{g}} e^{p / T}
$$

and the unitary relation $\left|\mathcal{M}_{12}\right|^{2}=\left|\mathcal{M}_{21}\right|^{2}$, Eq.(6) and Eq.(7) can be combined to give

$$
R_{g \rightarrow q}-R_{q \rightarrow g}=\left[\frac{1}{\lambda_{Q} \lambda_{\bar{Q}}}-\frac{1}{\lambda_{g}^{2}}\right] I_{\text {gluon }}
$$

where

$$
\begin{aligned}
I_{\text {gluon }}= & \frac{1}{2} \int \frac{d^{3} p_{1}}{(2 \pi)^{3} 2 E_{1}} \int \frac{d^{3} p_{2}}{(2 \pi)^{3} 2 E_{2}} \int \frac{d^{3} p_{3}}{(2 \pi)^{3} 2 E_{3}} \int \frac{d^{3} p_{4}}{(2 \pi)^{3} 2 E_{4}}(2 \pi)^{4} \delta^{4}\left(p_{1}+p_{2}-p_{3}-p_{4}\right) \times \\
& \sum\left|\mathcal{M}_{g g \rightarrow q \bar{q}}\right|^{2} f_{g}\left(p_{1}\right) f_{g}\left(p_{2}\right) f_{q}\left(p_{3}\right) f_{\bar{q}}\left(p_{4}\right) \times e^{\beta\left(E_{1}+E_{2}\right)}
\end{aligned}
$$

and $\beta^{-1}=T$. The above multi-dimensional integral can be reduced further by replacing

$$
\int \frac{d^{3} p}{2 E}=\int d^{4} p \delta\left(p^{2}-m^{2}\right) \theta\left(p_{0}\right)
$$

and by change of variables to:

$$
\begin{aligned}
q & =p_{1}+p_{2}, \\
p & =\frac{1}{2}\left(p_{1}-p_{2}\right), \\
q^{\prime} & =p_{3}+p_{4}, \\
p^{\prime} & =\frac{1}{2}\left(p_{3}-p_{4}\right) .
\end{aligned}
$$

One can easily eliminate the energy-momentum-conserving $\delta$ function by carrying out the integrals over $q^{\prime}$. Using spherical coordinates and defining $q$ along the z-axis where $q_{\mu}=$ $\left(q_{0}, 0,0, q\right), \quad p_{\mu}=\left(p_{0}, p \sin \theta, 0, p \cos \theta\right)$ and $p_{\mu}^{\prime}=\left(p_{0}^{\prime}, p^{\prime} \sin \phi \sin \chi, p^{\prime} \sin \phi \cos \chi, p^{\prime} \cos \phi\right)$, we get,

$$
\begin{aligned}
I_{\text {gluon }}= & \frac{1}{16(2 \pi)^{6}} \int_{0}^{\infty} d q_{0} \int_{0}^{\infty} d q \int_{-q_{0} / 2}^{q_{0} / 2} d p_{0} \int_{-q_{0} / 2}^{q_{0} / 2} d p_{0}^{\prime} \int_{0}^{\infty} d p \int_{0}^{\infty} d p^{\prime} \int_{-1}^{1} d(\cos \theta) \int_{-1}^{1} d(\cos \phi) \\
& \times \int_{0}^{2 \pi} d \chi \delta\left[p-\left[p_{0}^{2}+\frac{s}{4}\right]^{\frac{1}{2}}\right] \delta\left[p^{\prime}-\left[p_{0}^{\prime 2}-m^{2}+\frac{s}{4}\right]^{\frac{1}{2}}\right] \delta\left[\cos \theta-\frac{q_{0} p_{0}}{q p}\right] \delta\left[\cos \phi-\frac{q_{0} p_{0}^{\prime}}{q p^{\prime}}\right] \\
& \times \sum\left|\mathcal{M}_{g g \rightarrow q \bar{q}}\right|^{2} f_{g}\left[\frac{q_{0}}{2}+p_{0}\right] f_{g}\left[\frac{q_{0}}{2}-p_{0}\right] f_{q}\left[\frac{q_{0}}{2}+p_{0}^{\prime}\right] f_{\bar{q}}\left[\frac{q_{0}}{2}-p_{0}^{\prime}\right] e^{\beta q_{0}} .
\end{aligned}
$$

The summed squared matrix element for the process $g g \rightarrow q \bar{q}$, is given by [8] (where quarks are assumed to be massless)

$$
\sum\left|\mathcal{M}_{g g \rightarrow q \bar{q}}\right|^{2}=\gamma_{g}^{2} \gamma_{q} \gamma_{\bar{q}} \pi^{2} \alpha_{s}^{2}\left[\frac{u t}{3 s^{2}}+\frac{2}{27}\left(\frac{u}{t}+\frac{t}{u}\right)-\frac{1}{6}\right] .
$$

Here $\gamma_{g} \equiv 2\left(N_{c}^{2}-1\right)$ and $\gamma_{q}=\gamma_{\bar{q}} \equiv 2 N_{c} N_{f}$ are the number of internal degrees of freedom for gluons, quarks and anti-quarks respectively. $N_{c}=3$ is the number of colors and $N_{f}$ 
is the number of massless flavors. Throughout our analysis we have used $N_{f}=2.0$. The Mandelstam variables $s, t, u$ used in the above equations are given by

$$
\begin{aligned}
& s=q_{0}^{2}-q^{2} \\
& t=m^{2}-\frac{s}{2}\left(1-\frac{4 p_{0} p_{0}^{\prime}}{q^{2}}\right)+2\left[\frac{s}{4}\left(1-\frac{4 p_{0}^{2}}{q^{2}}\right)\right]^{\frac{1}{2}}\left[\frac{s}{4}\left(1-\frac{4 p_{0}^{\prime 2}}{q^{2}}\right)-m^{2}\right]^{\frac{1}{2}} \sin \chi \\
& u=-(s+t) .
\end{aligned}
$$

The matrix element given by Eq. (14) diverges in the limit $u, t \rightarrow 0$. Hence, the medium induced effective quark mass plays a crucial role and the divergence of the cross-section can be avoided by replacing 'm' with the thermal quark mass defined appropriately for a non equilibrium plasma 11, 12,

$$
m=m_{q}^{2}=\frac{g^{2}}{3 \pi^{2}} \int_{0}^{\infty} d p p\left[2 f_{g}+f_{q}+f_{\bar{q}}\right]
$$

The integrals over $p, p^{\prime}, \theta$ and $\phi$ of Eq. (13) have been performed carefully because of kinematical constraints to have the limits

$$
q_{0} \geq 2 m, \quad q^{2} \leq\left(q_{0}^{2}-4 m^{2}\right), \quad p_{0}^{2} \leq \frac{q^{2}}{4}, \quad p_{0}^{\prime 2} \leq \frac{q^{2}}{4}\left[1-\frac{4 m^{2}}{s}\right] .
$$

Thus we evaluate all but five of the integrals in Eq.(13) trivially 8] obtaining

$$
\begin{aligned}
I_{\text {gluon }}= & \frac{1}{16(2 \pi)^{6}} \int_{2 m}^{\infty} d q_{0} \int_{0}^{\left(q_{0}^{2}-4 m^{2}\right)^{\frac{1}{2}}} d q \int_{-p_{*}}^{p_{*}} d p_{0} \int_{-p_{*}^{\prime}}^{p_{*}^{\prime}} d p_{0}^{\prime} \\
& \times \int_{0}^{2 \pi} d \chi \sum\left|\mathcal{M}_{g g \rightarrow q \bar{q}}\right|^{2} f_{g}\left[\frac{q_{0}}{2}+p_{0}\right] f_{g}\left[\frac{q_{0}}{2}-p_{0}\right] f_{q}\left[\frac{q_{0}}{2}+p_{0}^{\prime}\right] f_{\bar{q}}\left[\frac{q_{0}}{2}-p_{0}^{\prime}\right] e^{\beta q_{0}}
\end{aligned}
$$

where the limits of integration are

$$
\begin{aligned}
& p_{*}=\frac{q}{2}, \\
& p_{*}^{\prime}=\frac{q}{2}\left(1-\frac{4 m^{2}}{s}\right)^{\frac{1}{2}} .
\end{aligned}
$$

Following Ref. [4], we write Eq.(9) in a convenient form

$$
R_{g \rightarrow q}-R_{q \rightarrow g}=n_{g} R_{2}\left(1-\frac{\lambda_{Q} \lambda_{\bar{Q}}}{\lambda_{g}^{2}}\right)
$$

where $n_{g}$ is the gluon density and $R_{2}$ is given by

$$
R_{2}=\frac{I_{\text {gluon }}}{\lambda_{Q} \lambda_{\bar{Q}} n_{g}}
$$




\section{FIGURES}
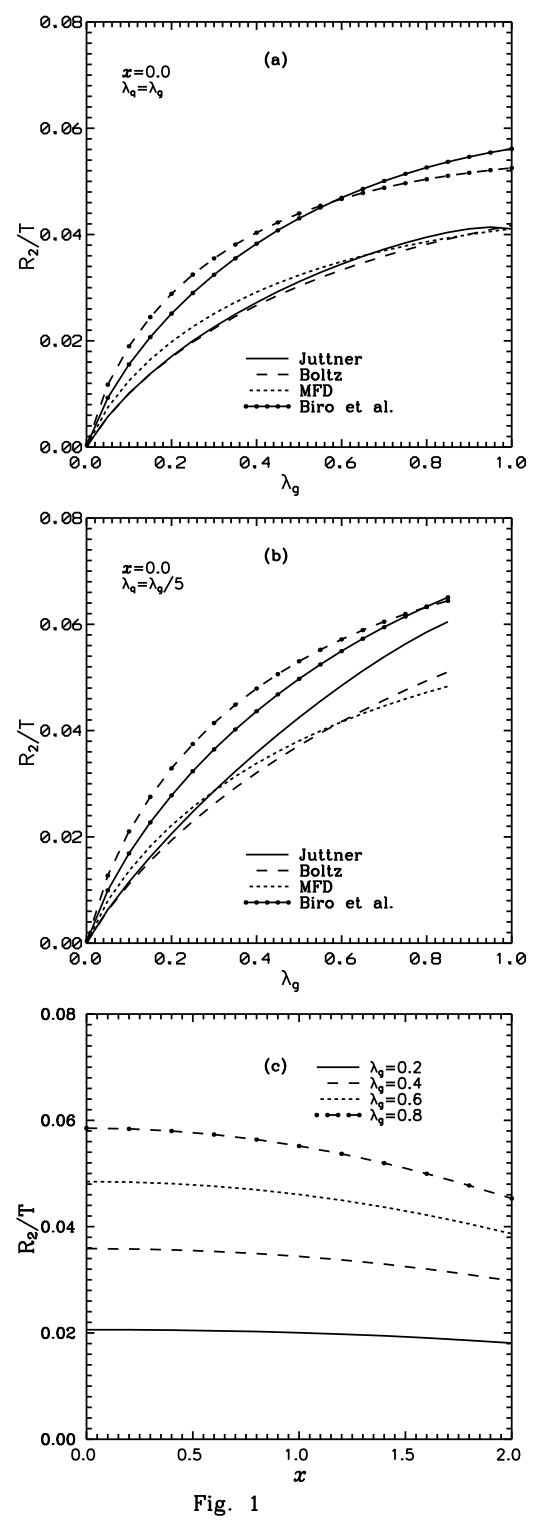

FIG. 1. (a)The quark production rate $R_{2} / T$ as a function of $\lambda_{g}\left(\lambda_{q}=\lambda_{\bar{q}}=\lambda_{g}\right)$ with Juttner, Boltzmann and MFD distribution function. for $x=0$. The solid dot curve is the approximate formula used by Biro et al.[4].The dashed dot curve is calculated rate with Boltzmann distribution function in the incoming channel and factorised distribution otherwise. (b)Same as (a) but with $\left(\lambda_{q}=\lambda_{\bar{q}}=\lambda_{g} / 5\right)$. (c) The quark production rate $R_{2} / T$ as a function of $x$ for different values of $\lambda_{g}$ where $\left(\lambda_{q}=\lambda_{\bar{q}}=\lambda_{g} / 5\right)$.

We compute $R_{2}$ numerically using Juttner, MFD and BM distribution functions and also compare the result with the approximation obtained by Biro et al 


$$
R_{2} \approx 0.24 N_{f} \alpha_{s}^{2} \lambda_{g} T \ln \left(\frac{1.65}{\alpha_{s} \lambda}\right)
$$

where $\alpha_{s}(=0.3)$ is the strong coupling constant. Note that the factor $\lambda=\left(\lambda_{g}+\cosh x \lambda_{q} / 2\right)$ which arises due to the thermal quark mass [7] is slightly different from what is used in Ref. [4]

Figs. 1(a) and 1(b) show $R_{2} / T$ as a function of $\lambda_{g}$ obtained numerically using Eq.(24) with Juttner, MFD and BM distribution functions at $\mathrm{T}=0.57 \mathrm{GeV}$. Initially, we consider a baryon free plasma $(x=0)$. In Fig. 1(a), we assume the gluon and quark (anti-quark) content of the plasma is equal $\left(\lambda_{q}=\lambda_{g}\right)$, whereas in Fig. 1(b), the plasma is assumed to be more gluon rich (say $\lambda_{q}=\lambda_{g} / 5$ ). As in Fig. 1(a), the $R_{2} / T$ values obtained using Juttner, MFD and BM distribution functions do not have significant difference at all values of $\lambda_{g}$. At small $\lambda_{g}, R_{2} / T$ obtained using MFD differs slightly which ultimately merges with that obtained using Juttner distribution function, as both Juttner and MFD distributions become equal when $\lambda_{q}=\lambda_{g}=1.0$ at $x=0$. However, when the plasma is gluon rich as in Fig. 1(b), $R_{2} / T$ values obtained with above three distributions do not differ much say up to $\lambda_{g} \approx 0.5$, beyond which the MFD and BM results show significant deviation. Recall that for $\lambda_{q}=\lambda_{g} / 5$, MFD and Juttner distributions (for $q$ and $\bar{q}$ ) are not equal when $\lambda_{g} \rightarrow 1$ even at $x=0$. We can conclude from these results that for unsaturated plasma, the $R_{2}$ values obtained using Juttner distribution do not deviate significantly from the results that can be obtained with the factorized distributions like MFD or BM functions. However, these results are quite less (20\% to $30 \%$ ) than what is found on the basis of the so called classical approximation i.e. using Eq.(25) given by Biro et al which are also shown in Figs. 1(a) and 1(b) for comparison. It is natural to think that the discrepancy found in Biro et al's estimation could be due to the classical aspect of the approximation which we investigate below.

In the classical approximation, Eq.(10) can be written as

$$
I_{\text {gluon }}=\frac{1}{2} \int \frac{d^{3} p_{1}}{(2 \pi)^{3}} \int \frac{d^{3} p_{2}}{(2 \pi)^{3}}\left[\sigma_{g g \rightarrow q \bar{q}} v_{12}\right] f_{g}\left(p_{1}\right) f_{g}\left(p_{2}\right)
$$

which represents the cross section for the process $g g \rightarrow q \bar{q}$ folded with the distributions for the initial particles. The cross section $\sigma_{g g \rightarrow q \bar{q}}$ is given by

$$
\sigma_{g g \rightarrow q \bar{q}}=\frac{1}{v_{12} 2 E_{1} 2 E_{2}} \int \frac{d^{3} p_{3}}{(2 \pi)^{3} 2 E_{3}} \int \frac{d^{3} p_{4}}{(2 \pi)^{3} 2 E_{4}}(2 \pi)^{4} \delta^{4}\left(p_{1}+p_{2}-p_{3}-p_{4}\right) \sum\left|\mathcal{M}_{g g \rightarrow q \bar{q}}\right|^{2}
$$

The classical approximation [7] assumes Boltzmann distribution function for quark, antiquark and gluon and also eliminates the Pauli blocking and Bose enhancement factors in 
the final states. The Eq.(26) can be factorized as $I_{\text {gluon }}=\bar{n}_{g}^{2} \sigma_{2} / 2$ where $\bar{n}_{g}$ is the equilibrium gluon density and $\sigma_{2}$ is a velocity weighted cross section. Defining $R_{2}=n_{g} \sigma_{2} / 2$, Biro et al estimate $\sigma_{2}, n_{g}$ and also thermal quark mass using factorized distribution functions ( same as Eqs. (3) and (4) with $x=0$ which become Fermi-Dirac and Bose-Einstein distributions in the limit $\left.\lambda_{i} \rightarrow 1.0\right)$. Within the present formalism, we can simulate the results of Biro et al by replacing $f_{q}$ and $f_{\bar{q}}$ in Eq.(20) with BM distribution (Eq. 5) and $f_{g}$ with Bose-Einstein type (Eq. 4). Also we estimate thermal quark mass using MFD type distributions as in Ref. [4] (i.e. using Eqs. (3) and (4) at $x=0$ ). The simulated result is also shown in Figs. 1(a) and 1(b) for comparison (see solid curve with dots). The results are in good agreement with that of Biro et al's estimation. Therefore, the deviation found using Eq.(25) is due to the use of FD and BE type of distribution functions instead of using BM functions which would have been appropriate under classical approximation. In fact, our results obtained from Eq.(20) using BM approximation is in a way classical. Further it may be mentioned here that the first term in the RHS of Eq.(9) arises due to the factors like Pauli blocking $\left(1-f_{q}\right)$ and Bose enhancement $\left(1+f_{g}\right)$. Only the integral $I_{\text {gluon }}$ is evaluated using various approximations. Therefore, even though we use the classical approximation like BM distribution, the final results still include the quantum effect. Similarly, Biro et al have estimated $I_{\text {gluon }}$ classically, but their final factorization does include quantum effect (see appendix B of Ref. [7] for detail). In other words, Biro et al approach will give correct estimation if BM approximation is used to estimate $R_{2}$. The above discussions confine only to baryon free plasma. Similar discrepancy is found at finite baryon density also.

Now we investigate the effect of baryon density on $R_{2}$. Fig. 1 (c) shows $R_{2} / T$ as a function of $x$ obtained numerically using actual (Juttner) distribution function at different values of fugacities where $\lambda_{q}=\lambda_{g} / 5$. It is interesting to note that $x$ dependence of $R_{2}$ is rather weak particularly when the plasma is unsaturated. The nearly $x$ independence is also evident from Eq. (1). For small values of $\lambda_{q}$, the contribution from the factor $\lambda_{q} e^{ \pm x}$ in the denominator is not very significant if $x$ is small. The $x$ dependence of the quark and anti-quark distribution functions mainly arises due to the $e^{ \pm x}$ factor in the numerator. Since these exponential factors get cancelled in the product, $f_{q} f_{\bar{q}}$ (hence $R_{2}$ ) will have weak $x$ dependence at small baryon density if the plasma is highly unsaturated.

\section{B. Gluon multiplication}

The rate $R_{3}$ for the process $g g \rightleftharpoons g g g$ depends on the differential radiative cross section (4.5.5 


$$
\frac{d \sigma_{3}}{d q_{\perp}^{2} d y d^{2} k_{\perp}}=\frac{d \sigma_{e l}^{g g}}{d q_{\perp}^{2}} \frac{d n_{g}}{d y d^{2} k_{\perp}} \theta\left(\lambda_{f}-\frac{\cosh y}{k_{\perp}}\right) \theta\left(\sqrt{s}-k_{\perp} \cosh y\right)
$$

where the first step function gives approximate LPM suppression factor and the second step function accounts for energy conservation, $s=18 T^{2}$ is the average squared center-of-mass energy of two gluons in the thermal gas. Here $k_{\perp}$ denotes the transverse momentum, $y$ is the longitudinal rapidity of the radiated gluon and $q_{\perp}$ denotes the momentum transfer in the elastic collision. The regularized gluon density distribution induced by a single scattering is

$$
\frac{d n_{g}}{d y d^{2} k_{\perp}}=\frac{C_{A} \alpha_{s}}{\pi^{2}} \frac{q_{\perp}^{2}}{k_{\perp}^{2}\left[\left(\mathbf{k}_{\perp}-\mathbf{q}_{\perp}\right)^{2}+m_{D}^{2}\right]} .
$$

Similarly the regularized small angle $g g$ scattering cross section is

$$
\frac{d \sigma_{e l}^{g g}}{d q_{\perp}^{2}}=\frac{9}{4} \frac{2 \pi \alpha_{s}^{2}}{\left(q_{\perp}^{2}+m_{D}^{2}\right)^{2}}
$$

where Debye screening mass $m_{D}$ given by

$$
m_{D}^{2}=\frac{3 g^{2}}{\pi^{2}} \int_{0}^{\infty} d p p\left[2 f_{g}+N_{f}\left(f_{q}+f_{\bar{q}}\right)\right]
$$

where $g^{2}=4 \pi \alpha_{s}$. The mean free path $\lambda_{f}$ for elastic scattering is then [5:7]

$$
\lambda_{f}^{-1}=\frac{9}{2} \pi \alpha_{s}^{2} n_{g} \frac{1}{m_{D}^{2}}\left[1+\frac{1}{\left(1+\frac{2}{9} \frac{m_{D}^{2}}{T^{2}}\right)}\right]
$$

Integrating $\phi$ part analytically

$$
\int_{0}^{2 \pi} d \phi \frac{1}{\left(\mathbf{k}_{\perp}-\mathbf{q}_{\perp}\right)^{2}+m_{D}^{2}}=\frac{2 \pi}{\sqrt{\left(k_{\perp}^{2}+q_{\perp}^{2}+m_{D}^{2}\right)^{2}-4 q_{\perp}^{2} k_{\perp}^{2}}},
$$

and defining $R_{3}=\frac{1}{2} n_{g} \sigma_{3}$, we can evaluate

$$
R_{3} / T=\frac{27 \alpha_{s}^{3}}{2} \lambda_{f}^{2} n_{g} I\left(\lambda_{g}, \lambda_{q}, x\right)
$$

where $I\left(\lambda_{g}, \lambda_{q}, x\right)$ is a function of $\lambda_{g}, \lambda_{q}$ and $x$,

$$
\begin{aligned}
I\left(\lambda_{g}, \lambda_{q}, x\right)= & \int_{1}^{\sqrt{s} \lambda_{f}} d x \int_{0}^{s / 4 m_{D}^{2}} d z \frac{z}{(1+z)^{2}} \\
& {\left[\frac{\cosh ^{-1}(\sqrt{x})}{x \sqrt{\left[x+(1+z) x_{D}\right]^{2}-4 x z x_{D}}}+\frac{1}{s \lambda_{f}^{2}} \frac{\cosh ^{-1}(\sqrt{x})}{\sqrt{\left[1+x(1+z) y_{D}\right]^{2}-4 x z y_{D}}}\right] }
\end{aligned}
$$

with $x_{D}=m_{D}^{2} \lambda_{f}$ and $y_{D}=m_{D}^{2} / s$.

As mentioned before, $R_{3}$ depends on baryo-chemical potential $\mu$ through the Debye screening mass $m_{D}$ which we evaluate using Juttner, BM and MFD distributions. 

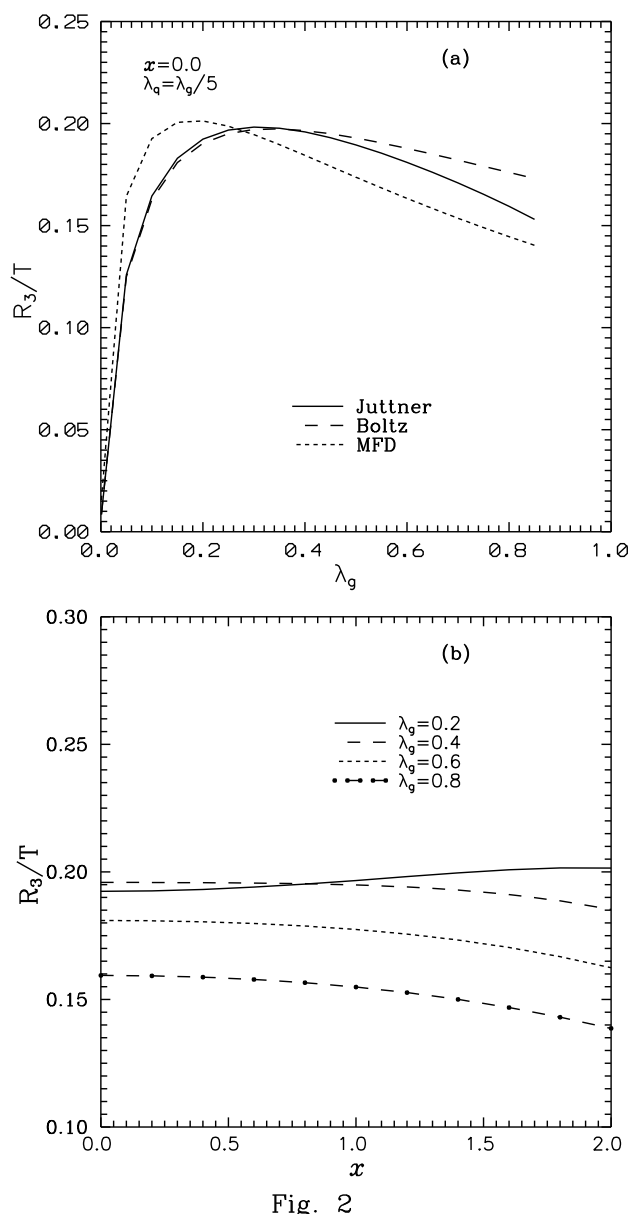

FIG. 2. (a)The gluon production rate, $R_{3} / T$ as function of $\lambda_{g}\left(\lambda_{q}=\lambda_{\bar{q}}=\lambda_{g} / 5\right)$ with Juttner, Boltzmann and MFD distribution function. for $x=0$. (b) The gluon production rate $R_{3} / T$ as a function of $x$ for different values of $\lambda_{g}$ where $\left(\lambda_{q}=\lambda_{\bar{q}}=\lambda_{g} / 5\right)$.

Fig. 2(a) shows $R_{3} / T$ as a function of $\lambda_{g}$ for a typical gluon rich plasma. At small values of $\lambda_{g}, R_{3} / T$ is not much sensitive on various distribution functions, but they start deviating when $\lambda_{g}$ exceeds $\approx 0.5$. Since the plasma remains highly unsaturated by the time $T$ drops to $T_{c}$, results based on MFD or BM distribution will not differ much from the actual result. Fig. 3(b) shows the variation of $R_{3} / T$ with $x$ at few typical values of $\lambda_{g}$. Again, the $x$ dependence on $R_{3}$ is found to be insignificant particularly at small baryon density.

\section{Chemical equilibration}

The space time evolution of the QGP is studied using ideal hydrodynamics in $(1+1)$ dimension along with the following master rate equations [4]

$$
\frac{\partial n_{g}}{\partial \tau}=\left(R_{2 \rightarrow 3}-R_{3 \rightarrow 2}\right)-\left(R_{g \rightarrow q}-R_{q \rightarrow g}\right)
$$




$$
\frac{\partial n_{q}}{\partial \tau}=\frac{\partial n_{\bar{q}}}{\partial \tau}=\left(R_{g \rightarrow q}-R_{q \rightarrow g}\right)
$$

where $R_{2 \rightarrow 3}$ and $R_{3 \rightarrow 2}$ denote the rates for the process $g g \rightarrow g g g$ and its reverse and $R_{g \rightarrow q}$ and $R_{q \rightarrow g}$ for the process $g g \rightarrow q \bar{q}$ and its reverse respectively. Similarly, the equation for the conservation of energy and momentum in $(1+1)$ dimension can be written as

$$
\frac{\partial \epsilon}{\partial \tau}+\frac{\epsilon+p}{\tau}=0
$$

which does not include viscosity effect [13]. In case of an ideal fluid, $3 p=\epsilon$. From the conservation of baryon number, one gets $\partial_{\mu}\left(n_{B} u^{\mu}\right)=0$ which results in

$$
n_{B} \tau=\left(n_{q}-n_{\bar{q}}\right) \tau=\text { const }
$$

We evaluate the RHS of Eq.(36) and Eq.(37) numerically using Juttner distribution. However, in order to solve the above coupled equations, we use factorized density distributions $n_{g}=\lambda_{g} \bar{n}_{g}$ and $n_{q(\bar{q})}=\lambda_{Q(\bar{Q})} \bar{n}_{q(\bar{q})}$ (where $\bar{n}_{i}$ are the equilibrium parton densities) obtained on the basis of Eqs. (3) and (4). Since the plasma is unsaturated, we could have also used the BM approximation (Eq. 5). But, we prefer to use Eqs. (3) and (4) for consistency with earlier works [4, []].

Finally, we solve the following set of equations for $\lambda_{g}, \lambda_{Q}, \lambda_{\bar{Q}}$ and $T$ numerically by using fourth order Runga-Kutta method [7],

$$
\begin{gathered}
\frac{\dot{\lambda}_{g}}{\lambda_{g}}+\frac{3 \dot{T}}{T}+\frac{1}{\tau}=R_{3}\left(1-\lambda_{g}\right)-2 R_{2}\left(1-\frac{\lambda_{Q} \lambda_{\bar{Q}}}{\lambda_{g}^{2}}\right), \\
\frac{\dot{\lambda}_{Q}}{\lambda_{Q}}+\frac{3 \dot{T}}{T}+\frac{1}{\tau}=R_{2} \frac{a_{1}}{b_{1}} \frac{\lambda_{g}}{\lambda_{Q}}\left(1-\frac{\lambda_{Q} \lambda_{\bar{Q}}}{\lambda_{g}^{2}}\right), \\
\dot{\lambda}_{\bar{Q}}=\dot{\lambda}_{Q}+\frac{\lambda_{Q}-\lambda_{\bar{Q}}}{\tau}+\frac{3 \dot{T}}{T}\left(\lambda_{Q}-\lambda_{\bar{Q}}\right), \\
\frac{3 \dot{T}}{T}+\frac{1}{\tau}=-\frac{3}{4 A_{t}}\left[a_{2} \dot{\lambda}_{g}+b_{2} \dot{\lambda}_{Q}+b_{2} \dot{\lambda}_{\bar{Q}}\right]
\end{gathered}
$$

where

$$
A_{t}=a_{2} \lambda_{g}+b_{2}\left(\lambda_{Q}+\lambda_{\bar{Q}}\right) .
$$

As in Ref. [5.7], we take the initial conditions as $T_{0}=0.57 \mathrm{GeV}, \lambda_{g 0}=0.09, \lambda_{q 0}=0.02$ at $\tau_{0}=0.3 \mathrm{fm} / \mathrm{c}$ and treat $x_{0}(\mu / T)$ as a parameter. 

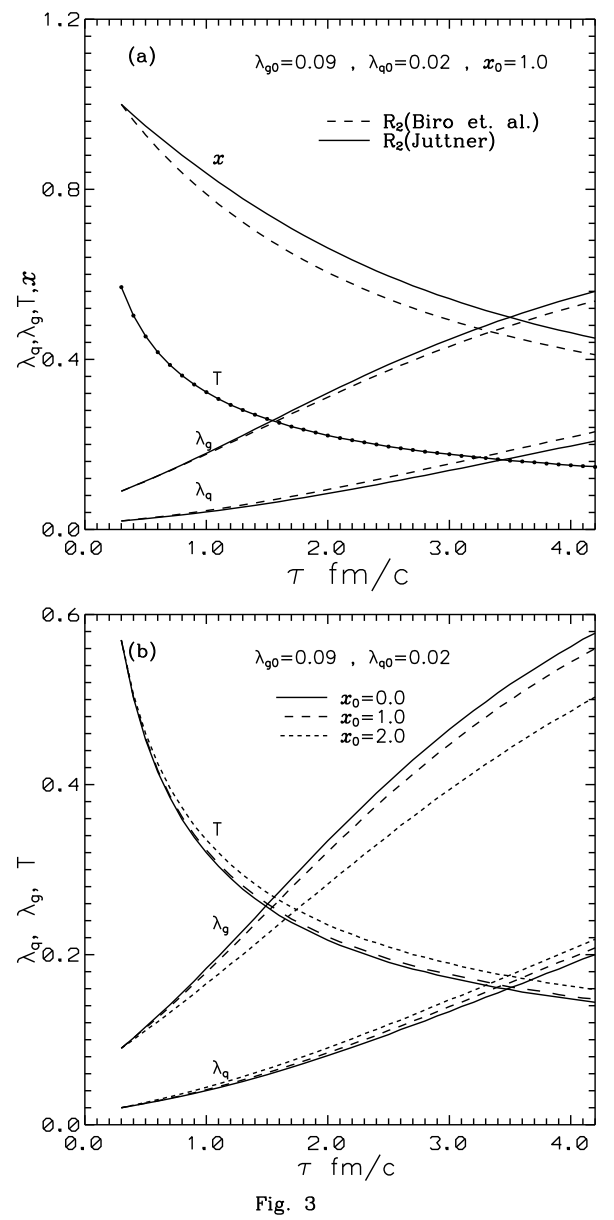

FIG. 3. (a) The temperature, the quark chemical potential $x=\mu / T$, the gluon fugacity $\lambda_{g}$, the quark fugacity $\lambda_{q}$, as a function of $\tau$ for $R_{2}$ integrated using Juttner distribution and also for $R_{2}$ using approximate formula of Biro et al. [4] with the initial conditions $T_{0}=0.57 \mathrm{GeV}$, $\lambda_{g 0}=0.09$ and $\lambda_{q 0}=0.02, x_{0}=1.0$. (b)The time dependence of $T, x, \lambda_{g}$ and $\lambda_{q}$ with initial conditions $T_{0}=0.57 \mathrm{GeV}, \lambda_{g 0}=0.09$ and $\lambda_{q 0}=0.02$ for $x=0.0,1.0,2.0$.

Fig. 3(a) shows temperature $\mathrm{T}$ and the fugacities as function of $\tau$ obtained with the revised values of $R_{2}$ and $R_{3}$ (solid curves). The dashed curves are obtained using Biro et al's factorization. Since $R_{2}$ values are found less as compared to Biro et al's result, the equilibration rate for $\lambda_{q}$ slows down and the rate for $\lambda_{g}$ goes up marginally at large $\tau$. The equilibration rate for $x$ also slows down. This implies that the rate of decrease (increase) of quark (anti-quark) contents becomes slower. However, as increase in the rate for $\lambda_{g}$ and decrease in the rate of $\lambda_{q}$ have opposite effects on temperature, the variation of $\mathrm{T}$ with $\tau$ remains practically unchanged. In Fig. 3(b), we show the effect of baryon density on chemical equilibration with revised rates $R_{2}$ and $R_{3}$. As before [0], the gluon equilibration rate slows down much more than the slight enhancement found in quark (antiquark) equilibration rate. The overall effect is that the temperature of the plasma falls at a 
slower rate as compared to the plasma when it is baryon free.

\section{THERMAL HARD PHOTON PRODUCTION}

Hard photons are the promising probes to study the evolution of the quark gluon plasma produced in relativistic heavy ion collisions. So far photon emission has been considered mostly from either chemically equilibrated or non-equilibrated plasma at zero baryon density [14 [16]. In Ref. [17,18], hard photon yield has been calculated from a chemically equilibrated baryon rich plasma. Here we investigate the photon production rate and integrated yields from a chemically unsaturated plasma at finite baryon density.

In a thermodynamic system, the photon production rate and its momentum distribution depend on the momentum distribution of the quarks, anti-quarks and the gluons, which is governed by the thermodynamic state of the plasma. To get the emission rate of a photon from the plasma we have to fold the amplitude for these reactions with the thermal distribution functions and integrate over the phase space volume of all particles except photon. The calculation of photon emission rate from a QGP follows via two steps. The emission rate from a stationary plasma at a fixed temperature $\mathrm{T}$ is determined using Juttner distribution function and the yield is calculated by integrating over the plasma volume created by the expansion and consequent cooling of the plasma. In thermal and chemical equilibrium, the production rate of hard photons with energy $E \gg T$ can be computed using the Braaten-Yaan prescription [19]. Then the rate decomposes into a soft part, which is treated using a resummed quark propagator according to Braaten-Pisarsky Method [20], and a hard part containing only bare propagators and vertices. The medium effects in the QGP are included in the soft part through the thermal quark mass, which serves as an infrared cutoff in the case of a vanishing bare quark mass. The hard part follows from the momentum integration over the matrix elements that lead to photon emission in lowest order multiplied by the distribution functions of the incoming and outgoing partons [14]. A separation scale $k_{c}$ is introduced, which allows one to distinguish between soft and hard momenta of the intermediate quark.

In the following, we only consider the hard part of the photon emission rate and the cut-off parameter $k_{c}^{2}$ is replaced by the thermal mass $2 m_{q}^{2}$. The main processes of photon production are $\left(\mathrm{O}\left(\alpha \alpha_{s}\right)\right.$ annihilation $q \bar{q} \rightarrow g \gamma$ and Compton processes $\left.q(\bar{q}) g \rightarrow q(\bar{q}) \gamma\right)$. The thermal rates for these reactions are

$$
2 E \frac{d R^{\text {hard }}}{d^{3} p}=\frac{N}{(2 \pi)^{8}} \int \frac{d^{3} p_{1}}{2 E_{1}} \frac{d^{3} p_{2}}{2 E_{2}} \frac{d^{3} p_{3}}{2 E_{3}} f_{1}\left(E_{1}\right) f_{2}\left(E_{2}\right)\left(1 \pm f_{3}\left(E_{3}\right)\right)
$$




$$
\delta\left(p_{1}^{\mu}+p_{2}^{\mu}-p_{3}^{\mu}-p^{\mu}\right) \sum|\mathcal{M}|^{2}
$$

where $f_{1,2,3}$ are the parton distribution function corresponding to two processes [7], with plus sign for annihilation and the minus sign for the two Compton processes. Following Ref. 14, 18, the above equation can be written as

$$
\begin{aligned}
2 E \frac{d R^{\text {hard }}}{d^{3} p}= & \frac{1}{8(2 \pi)^{7} E} \int_{2 k_{c}^{2}}^{\infty} d s \int_{-s+k_{c}^{2}}^{-k_{c}^{2}} d t \sum|\mathcal{M}|^{2} \\
& \times \int_{R^{2}} d E_{1} d E_{2} \frac{\Theta\left(P\left(E_{1}, E_{2}\right)\right) f_{1} f_{2}\left(1 \pm f_{3}\right)}{\sqrt{P\left(E_{1}, E_{2}\right)}}
\end{aligned}
$$

where $P\left(E_{1}, E_{2}\right)=a E_{2}^{2}+b E_{2}+c$, with a,b,c as given by

$$
a=-(s+t)^{2} ; b=2(s+t)\left(E s-E_{2} t\right) ; c=s t(s+t)-\left(E_{s}+E_{2}\right)^{2}
$$

and $\Theta$ is the step function, $s, t, u$ are the Mandelstam variables. The matrix elements for the Compton and annihilation processes can be written as

$$
\begin{aligned}
& \sum|\mathcal{M}|^{2}=\frac{2^{9} 5}{9} \pi^{2} \alpha \alpha_{s} \frac{u^{2}+t^{2}}{u t} \\
& \sum|\mathcal{M}|^{2}=-\frac{2^{9} 5}{9} \pi^{2} \alpha \alpha_{s} \frac{s^{2}+t^{2}}{s t}
\end{aligned}
$$

For the case $\mu=0$, it is possible to obtain an approximate analytic expression for Eq.(45) assuming BM distribution for $f_{1}$ and $f_{2}$ and full Fermi/Bose distribution functions for $f_{3}$ [14]. Since we consider baryon rich unsaturated plasma and use Juttner distribution function, we have to employ numerical methods. Following [18], we rewrite Eq.(45) in a form suitable for Gauss quadrature with $E_{+}:=E_{1}+E_{2}$

$$
\begin{aligned}
& 2 E \frac{d R^{\text {hard }}}{d^{3} p}=\frac{5 \alpha \alpha_{s}}{18 \pi^{5} E} e^{-E / T-k_{c}^{2} / 2 E T} \underbrace{\int_{2 k_{c}^{2}}^{\infty} d s e^{-\left(s-2 k_{c}^{2}\right) / 4 E T}}_{\text {Lagauerre }} \frac{1}{s} \underbrace{\int_{-s+k_{c}^{2}}^{-k_{c}^{2}}}_{\text {Legendre }} d t|\mathcal{M}(s, t)|^{2} \\
& \underbrace{\int_{E+\frac{s}{4 E}}^{\infty} d E_{+} e^{-\left(E_{+}-E-\frac{s}{4 E}\right) / T}}_{\text {Lagauerre }} \cdot \frac{1}{1 \mp \lambda_{3} e^{-\left(E_{+}-E\right) / T}} \\
& \underbrace{\int_{E_{2}^{-}}^{E_{2}^{+}} \frac{d E_{2}}{\sqrt{P_{1}\left(E_{+}, E_{2}\right)}}}_{\text {Chebyshev }} \frac{\lambda_{1}}{1 \pm \lambda_{1} e^{-\left(E_{+}-E_{2}\right) / T}} \frac{\lambda_{2}}{1+\lambda_{2} e^{-E_{2} / T}}
\end{aligned}
$$

where the upper signs and $\lambda_{1}=\lambda_{Q}, \lambda_{2}=\lambda_{\bar{Q}}, \lambda_{3}=\lambda_{g}$, is used for annihilation process, the Compton processes require lower signs and $\lambda_{1}=\lambda_{g}, \lambda_{2}=\lambda_{3}=\lambda_{Q(\bar{Q})}$ and the two results for $\lambda_{Q}$ and $\lambda_{\bar{Q}}$ are to be added in order to take quarks and anti-quarks into consideration. 
The polynomial $P_{1}\left(E_{+}, E_{2}\right)$ in Eq.(48) is $P\left(E_{+}-E_{2}, E_{2}\right) / s^{2}$ and has proper weight for Gauss-Chebyshev quadrature in $E_{2}$, where $E_{2}^{-}$and $E_{2}^{+}$are the two roots of the polynomial $P\left(E_{+}, E_{2}\right)$. The $E_{+}$integral as well as the s integral, is done numerically by Gauss-Laguerre quadrature and the $t$ integral is performed using Gauss-Legendre quadrature. This way, the accuracy of the integral has been improved. We have also verified the result using BM distribution for $f_{1}$ and $f_{2}$ that matches with the analytic results corresponding to equilibrated plasma at $x=0$ in the limit $k_{c}^{2} \rightarrow 0$.
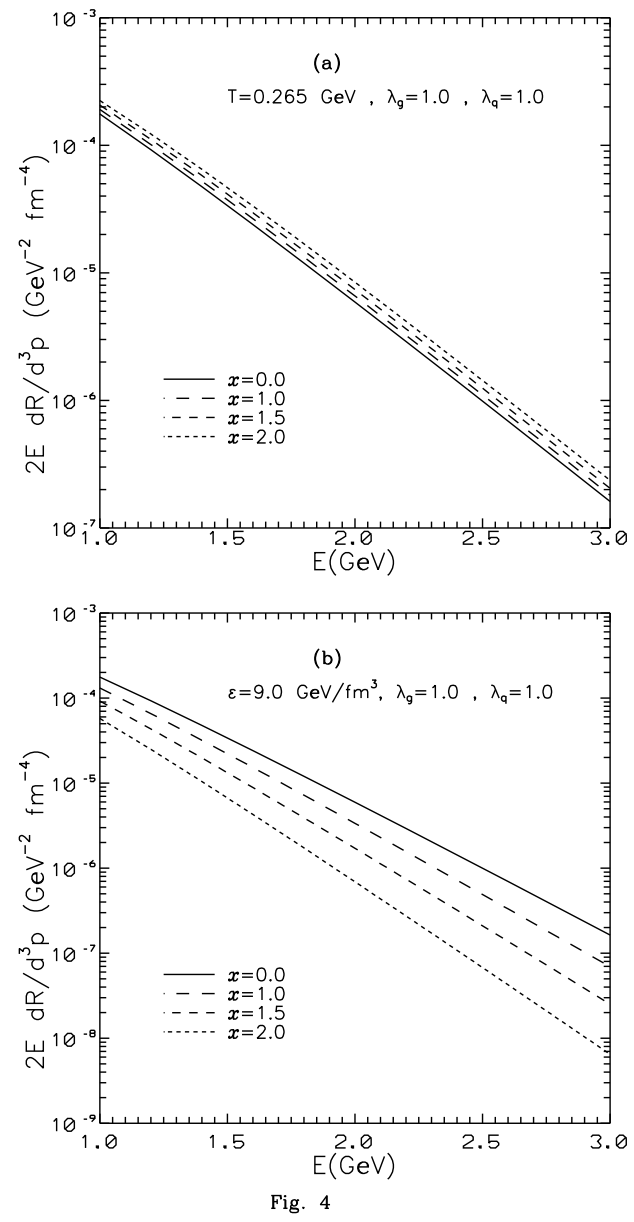

FIG. 4. (a) The photon production rate $2 E d R / d^{3} p$ for a chemically equilibrated plasma as a function of photon energy $\mathrm{E}$ at a fixed initial temperature $T_{0}=0.265 \mathrm{GeV}$ corresponds to energy density $\epsilon=9.0 \mathrm{GeV} / \mathrm{fm}^{3}$ when $x=0$ for $x=0.0,1.0,1.5$ and 2.0 and $\lambda_{g}=\lambda_{q}=1.0$. (b) The photon production rate $2 E d R / d^{3} p$ as a function of photon energy $\mathrm{E}$ at a fixed initial energy density $\epsilon_{0}=9.0 \mathrm{GeV} / \mathrm{fm}^{3}$ for $x=0.0,1.0,1.5$ and 2.0 and $\lambda_{g}=\lambda_{q}=1.0$. 
Figs. 4(a) and 4(b) show the photon production rate for a chemically equilibrated plasma both at a fixed temperature $(T=0.265 \mathrm{GeV})$ and fixed energy density $\left(\epsilon=9.0 \mathrm{GeV} / \mathrm{fm}^{3}\right)$. The energy density $\epsilon$ of $9.0 \mathrm{Gev} / \mathrm{fm}^{3}$ corresponds to an initial temperature of $0.265 \mathrm{GeV}$ at $x=0$. These results are similar to what was found in Ref. [18]. We have further extended the work to calculate the photon production from unsaturated plasma at finite baryon density. Figs. 5(a) and 5(b) shows the corresponding results for an unsaturated plasma. The fugacities shown in the figure caption correspond to an initial temperature of $0.57 \mathrm{GeV}$ for energy density $\epsilon=9.0 \mathrm{GeV} / \mathrm{fm}^{3}$ at $x=0$. Both Figs. 4(a) and 5(a) show similar behaviour namely, that the rate of photon production is enhanced with increasing baryon density. As will be shown below with analytic expression, the annihilation part is not affected much by $x$ whereas the Compton process for quark(anti-quark) is enhanced (suppressed) exponentially $\left(e^{ \pm x}\right)$. Therefore, the enhancement with increasing baryon density comes due to the quark part of the Compton process. At fixed energy density, the temperature also decreases with the increasing baryon density. Hence the net effect is that the photon rate decreases with increasing $x$ if the plasma is saturated (see Fig. 4(b). In the case of unsaturated plasma, the decrease of initial temperature with increasing $x$ is not very significant to compensate the Compton enhancement. The overall effect is, therefore, that the rate still increases with increasing $x$.

Since we are mainly interested to calculate the integrated photon yield which involves a six-dimensional integration (see Eq. 52 below) and will be time consuming, we look for an approximate analytic solution for photon production rate i.e. an approximation to Eq.(45). For a chemically unsaturated plasma, we can use MFD distribution functions to factorize the product appearing in Eq. (44) as

$$
f_{1} f_{2}\left(1 \pm f_{3}\right)=\lambda_{1} \lambda_{2} \lambda_{3} f_{1}^{e q} f_{2}^{e q}\left(1 \pm f_{3}^{e q}\right)+\lambda_{1} \lambda_{2}\left(1-\lambda_{3}\right) f_{1}^{e q} f_{2}^{e q}
$$

The factorization is similar to what has been used in Ref. [16] to estimate the photon yield from a baryon free unsaturated plasma except that the fugacities $\lambda_{1}, \lambda_{2}, \lambda_{3}$ are replaced by $\lambda_{g}, \lambda_{Q}, \lambda_{\bar{Q}}$ corresponding to gluon, quark and anti-quark respectively. The photon production rate can now be written in two parts. 

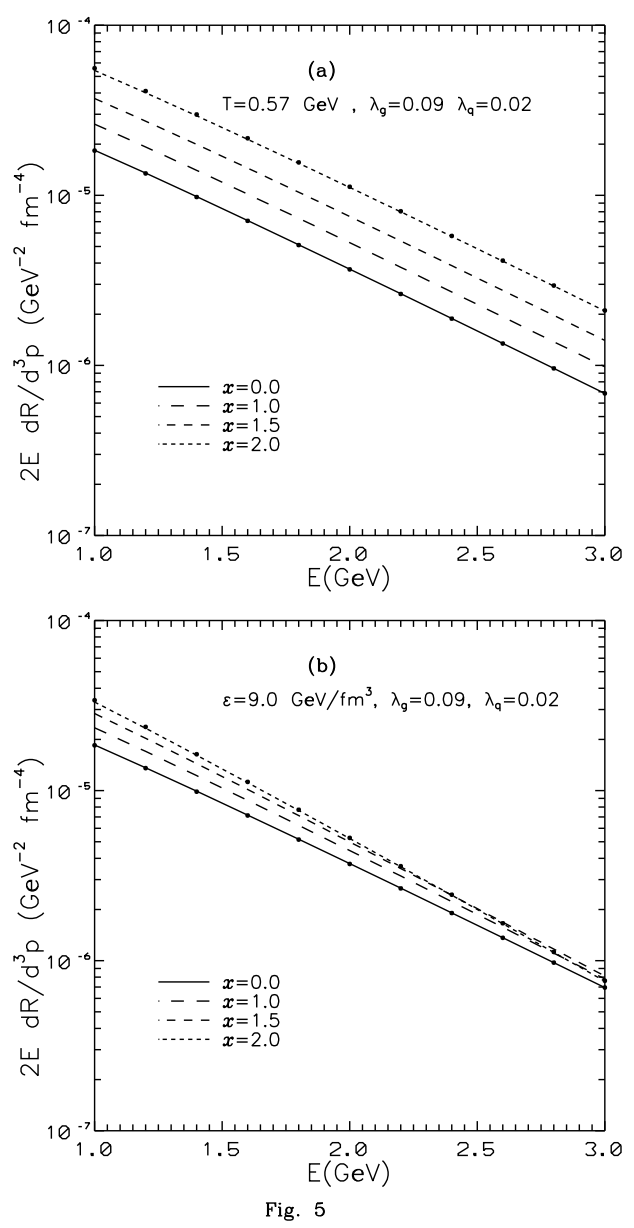

FIG. 5. (a) The photon production rate $2 E d R / d^{3} p$ chemically non-equilibrated plasma as a function of photon energy $\mathrm{E}$ at a fixed initial temperature $T_{0}=0.265 \mathrm{GeV}$ for $x=0.0,1.0,1.5$ and 2.0 and $\lambda_{g}=.09, \lambda_{q}=.02$. The dots are the results of the approximate formula (sum of Eq.(51) Eq. (52)). (b) The photon production rate $2 E d R / d^{3} p$ as a function of photon energy $\mathrm{E}$ at a fixed initial energy density $\epsilon_{0}=9.0 \mathrm{GeV} / \mathrm{fm}^{3}$ for $x=0.0,1.0,1.5$ and 2.0 and $\lambda_{g}=.09, \lambda_{q}=1.0$. The dots are the results of the approximate formula (sum of Eq.(51) and Eq. (52)).

For the first part, one can use the analytic form that can be obtained using BM distribution for $f_{1}$ and $f_{2}$ as in the case for equilibrated baryon free plasma [14] and given by

$$
\begin{aligned}
\left(2 E \frac{d R}{d^{3} p}\right)_{1}= & \frac{5 \alpha \alpha_{s}}{9 \pi^{2}} T^{2} e^{-E / T}[\underbrace{\lambda_{Q} \lambda_{\bar{Q}} \lambda_{g}\left\{\frac{2}{3} \ln \left(\frac{4 E T}{k_{c}^{2}}\right)-1.43\right\}}_{\text {annihilation }} \\
& +\underbrace{\left(\lambda_{Q}^{2} \lambda_{g}+\lambda_{\bar{Q}}^{2} \lambda_{g}\right)\left\{\frac{1}{6} \ln \left(\frac{4 E T}{k_{c}^{2}}\right)+0.0075\right\}}_{\text {Compton }}]
\end{aligned}
$$

Following Ref. [16], the second part can be written as 


$$
\begin{aligned}
\left(2 E \frac{d R}{d^{3} p}\right)_{2}= & \frac{10 \alpha \alpha_{s}}{9 \pi^{4}} T^{2} e^{-E / T}[\underbrace{\lambda_{Q} \lambda_{\bar{Q}}\left(1-\lambda_{g}\right)\left\{-2-2 \gamma+2 \ln \left(\frac{4 E T}{k_{c}^{2}}\right)\right\}}_{\text {annihilation }} \\
& +\underbrace{\frac{1}{2}\left\{\lambda_{Q} \lambda_{g}\left(1-\lambda_{Q}\right)+\lambda_{\bar{Q}} \lambda_{g}\left(1-\lambda_{\bar{Q}}\right)\right\}\left\{1-2 \gamma+2 \ln \left(\frac{4 E T}{k_{c}^{2}}\right)\right\}}_{\text {Compton }}]
\end{aligned}
$$

where $\alpha, \alpha_{s}$ are electromagnetic and strong coupling constants respectively. In the above, the first term is the contribution from the annihilation whereas the second and third terms are due to Compton like processes. The soft part of the contribution is taken care by choosing the cut-off parameter $k_{c}^{2}=2 m_{q}^{2}$. As argued before, the annihilation part is nearly independent of $x$ (the dependence on $\mu$ through $k_{c}^{2}$ is logarithmic) where as the Compton like processes depend on $x$ directly through $\lambda_{Q}$ or $\lambda_{\bar{Q}}$. The photon rate is the sum of Eq.(50) and Eq.(51). This formula reproduces the results of photon production rate quite accurately (when the plasma is unsaturated) with the exact result obtained from numerical integration. The results are shown in Figs. 5(a) and 5(b) (see solid circles) for comparison at two values of $x=0$ and $x=2.0$. The above analytic expression also reproduces the result for saturated plasma although the agreement is not so exact as in case of Figs. 5(a) and 5(b).

Next we calculate the photon yield by integrating the above analytic expression of photon production rate (sum of Eq. (50) and Eq. (51)) over the space and time 16]

$$
\left(\frac{2 d n}{d^{2} p_{\perp} d y}\right)=\left.Q \int_{\tau_{0}}^{\tau_{c}} d \tau \tau \int_{-y_{n u c}}^{y_{n u c}} d y^{\prime}\left(2 E \frac{d n}{d^{3} p d^{4} x}\right)\right|_{E^{l o c} \text { rest }=p_{\perp} \cosh \left(y-y^{\prime}\right), T=T(\tau), \lambda=\lambda(\tau)}
$$

The $\tau$ dependence of $T(\tau), \lambda_{g}(\tau)$ and $\lambda_{Q(\bar{Q})}(\tau)$ are taken from the above calculations (in previous section) with $\alpha_{S}=0.3, \lambda_{g 0}=0.09, \lambda_{q 0}=0.02$ and $T_{0}=0.57 \mathrm{GeV}$. Further, we take $y_{\text {nuc }}= \pm 6$ corresponding to RHIC energy. The transverse cross-section of the gold nuclei, $Q \approx 180 \mathrm{fm}^{2}$. Figs. 6(a) and 6(b) show the above integrated yields both at a fixed initial temperature and fixed energy density. The integrated yields both at fixed temperature and fixed energy density behave similarly as the rates shown in Figs. 5(a) and 5(b). It may be pointed out that, the finite baryon density effects which result in a slower cooling of the plasma affect the thermal photon and dilepton production yields somewhat differently. For example the basic rate of thermal dilepton production (studied in previous work Ref. [7]) does not depend on $x$ as it involves only $\lambda_{Q} \lambda_{\bar{Q}}$. 

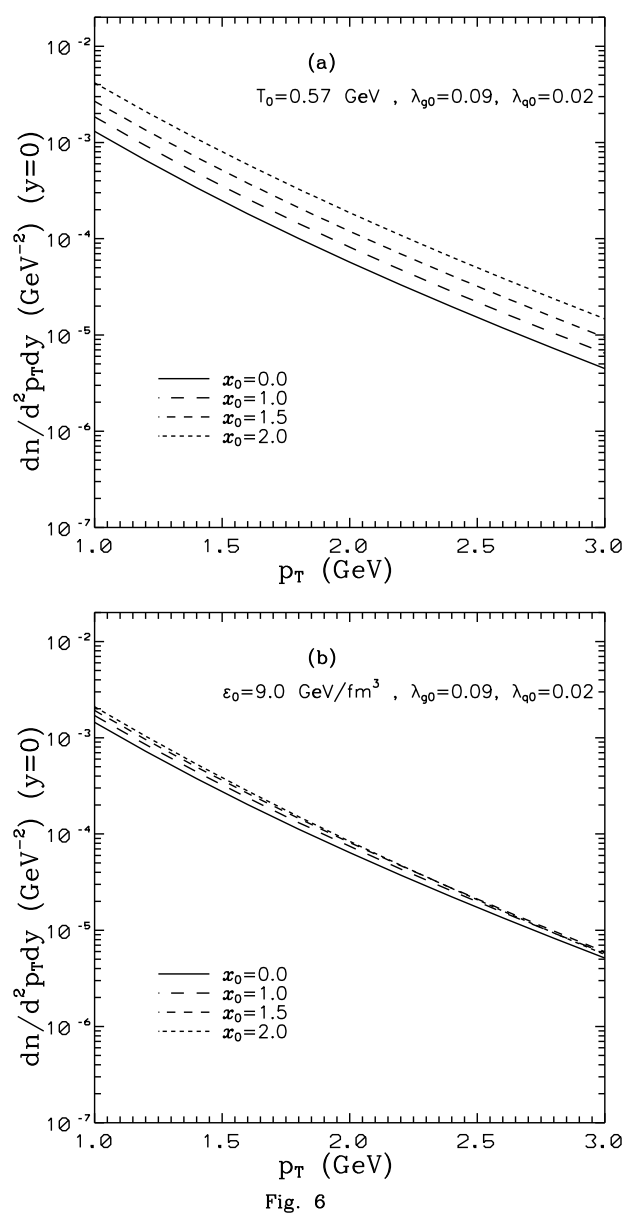

FIG. 6. (a) The integrated photon yield $d n / d_{T}^{p} d y$ from a chemically non-equilibrated plasma at a fixed initial temperature $T_{0}=0.57 \mathrm{GeV}$ for various values of $x_{0}$. (b) The integrated photon yield from a chemically non-equilibrated plasma at a fixed initial energy density $\epsilon_{0}=9.0 \mathrm{GeV} / \mathrm{fm}^{3}$ for various values of $x_{0}$.

But the integrated yield is enhanced due to the slower space time evolution at finite baryon density. On the other hand, in case of thermal photon production, the Compton like processes involve the product of the type $\lambda_{g} \lambda_{Q}$ or $\lambda_{g} \lambda_{\bar{Q}}$. Therefore, unlike the dilepton case, the basic photon yield also gets affected due to chemical equilibration since the variation of $x$ and $\lambda_{g}$ are significant.

\section{SUMMARY AND CONCLUSION}

In the present work, we have investigated the effect of baryon density on parton production for the process $g g \rightleftharpoons g g g$ and $g g \rightleftharpoons q \bar{q}$ with full phase space distribution and also including quantum effects like Pauli blocking and Bose enhancement factors explicitly. The results obtained using exact distribution function (Juttner) does not differ much from the 
results obtained using factorized distribution function of MFD or Boltzmann (BM) type. Although, BM approximation is classical, the final expression for the rate $g g \rightleftharpoons q \bar{q}$ includes quantum statistics. The results which are obtained on the basis of Biro et al's estimation, although classical in nature, overestimate the rate by $20 \%$ to $30 \%$ due to use of MFD type of approximation. Further it is found that, the rates for both the processes $(g g \rightleftharpoons g g g$ and $g g \rightleftharpoons q \bar{q})$ are insensitive to baryon density particularly when the plasma is unsaturated. This further justifies the use of MFD or BM type of distribution for partons for unsaturated plasma at small baryon density. We have also studied the process of chemical equilibration using the revised rates and also compare the results with the previous values obtained as per Biro et al's estimation. It is found, that the quark and gluon equilibration rates are affected slightly whereas the temperature remains unaffected. We have also studied thermal hard photon yields from a chemically non-equilibrated plasma which are considered to be the ideal probe to study the space time evolution of the plasma. We employ numerical techniques to estimate photon rate using Juttner distribution functions and also compare our results with approximate analytic expression. It is seen that as in the case of the dilepton yields, the photon yield is also enhanced in presence of finite baryon density. 


\section{REFERENCES}

[1] E. Shuryak, Phys. Rev. Lett. 68, 3270 (1992).

[2] J. D. Bjorken, Phys. Rev. D27, 140 (1983).

[3] K. Geiger and J. I. Kapusta, Phys. Rev. D47, 4905 (1993); K. Geiger and B. Muller, Nucl. Phys. 369, 600 (1992); K. Geiger, Physics Report, 258, 376 (1995); K. J. Eskola and X. N. Wang, Phys. Rev. D49, 1284 (1994).

[4] T.S. Biro, E. van Doorn, B. Muller, M. H. Thoma and X. N. Wang, Phys. Rev. C 34 1275 (1993).

[5] P. Levai, B. Muller and X. N. Wang, Phys. Rev. C51, 3326 (1995).

[6] D. K. Srivastava, M. G. Mustafa and B. Muller, Phys. Lett. B396, 45, (1997); Phys. Rev. C56, 1064 (1997).

[7] D. Dutta, K. Kumar, A. K. Mohanty and R K Choudhury, Phys.Rev. C60, 014905 (1999).

[8] T. Matsui, B. Svetitsky and L. McLerran, Phys. Rev. D34, 783 (1986).

[9] T. Altherr and D. Seibert, Phys. Rev. C49, 1684 (1994).

[10] D. Kharzeev, Phys. Lett. B378, 238 (1996); S. E. Vance, M. Gyulassy and X. N. Wang, Nucl. Phys. A638, 395c (1998).

[11] H. Vija and M. H. Thoma, Phys. Lett. B346, 329 (1995).

[12] R. Baier, M. Dirks, K. Redlich and D. Schiff, Phys. Rev. D56, 2548 (1997)

[13] P. Danielewicz and M. Gyulassy, Phys. Rev. D31, 53 (1985)

[14] J. Kapusta, P. Lichard and D. Seibert, Phys. Rev. D44, 1298 (1991); S. Chakrabarty, J. Alam, D. K. Srivastava and B. Sinha, Phys. Rev. D46, 3802 (1992); D. K. Srivastava and B. Sinha, Phys. Rev. Lett. 73, 2421 (1994); J. Alam, S. Raha and B. Sinha, Phys. Rep. 273, 243 (1996).

[15] M. Strickland, Phys. Lett. B331, 1994, 245.

[16] C. T. Traxler and M. H. Thoma, Phys. Rev. C53, 1348 (1996).

[17] A. Dumitru, D. H. Rischke, H. Stocker and W. Greiner, Mod. Phys. Lett. A8, 1291 (1993). 
[18] C. T. Traxler, H. Vija and M. H. Thoma, Phys. Lett. B346, 329 (1996).

[19] E. Braaten and T. C. Yuan, Phys. Rev. Lett. 66, 2183 (1991).

[20] E. Braaten and R. Pisarski, Nucl. Phys. B337, 569 (1990); B339, 310 (1990). 